\title{
Measurement of target-polarization dependent azimuthal asymmetries in SIDIS and Drell-Yan processes at COMPASS experiment
}

\author{
Bakur Parsamyan* \\ CERN, University of Turin and INFN section of Turin \\ E-mail: bakur.parsamyanecern.ch
}

\begin{abstract}
According to QCD the quark structure of the nucleon spin within the twist-2 approximation is parameterized by eight transverse-momentum-dependent (TMD) parton distribution functions (PDFs). The PDFs describe the distributions of longitudinal and transverse momenta of partons and their correlations with nucleon and quark polarizations and can be accessed through measurement of spin (in)dependent azimuthal asymmetries in semi-inclusive DIS (SIDIS) and Drell-Yan processes. The COMPASS experiment at CERN, as part of its programme, addresses the exploration of the spin structure of the nucleon by measuring spin (in)dependent azimuthal asymmetries in semi-inclusive DIS and, recently, also in Drell-Yan processes. Between 2002 and 2011 COMPASS performed a series of SIDIS measurements, using a longitudinally polarized muon beam impinging on longitudinally and transversely polarized ${ }^{6} \mathrm{LiD}$ or $\mathrm{NH}_{3}$ targets. Drell-Yan measurements with a $\pi^{-}$beam interacting with a transversely polarized $\mathrm{NH}_{3}$ target started with the 2015 run and will be continued in 2018. The measurement of the Sivers and other azimuthal asymmetries at practically the same hard scale in polarized SIDIS and Drell-Yan provides a unique possibility to test predicted in QCD (pseudo-)universal features of transversemomentum-dependent parton distribution functions.
\end{abstract}

The main focus of this review is set on the first ever polarized Drell-Yan measurements performed by COMPASS and on the recent results obtained for target longitudinal and transverse spin dependent SIDIS asymmetries.

QCD Evolution 2017

22-26 May, 2017

Jefferson Lab Newport News, VA - USA

${ }^{*}$ Speaker. 


\section{Introduction}

Within QCD quark transverse-momentum-dependent (TMD) parton distribution functions (PDFs) provide a three-dimensional picture of a fast moving nucleon in momentum space and play an important role in the theoretical description of high energy reactions. At leading order (LO) of QCD parton model the spin and quark-transverse-momentum structure of the nucleon is fully described by six time reversal even and two time reversal odd twist-2 TMD PDFs [1, 2]. Going beyond the leading order approximation, sixteen twist-3 PDFs related to quark-gluon correlations have to be introduced already at the first sub-leading accuracy level [2]. The TMD PDFs are universal, process-independent functions ${ }^{1}$ [3] describing longitudinal and transverse momenta distributions of partons and their correlations with nucleon and quark spins. Such correlations induce azimuthal modulations (asymmetries) in the cross sections of SIDIS ( $\ell N \rightarrow \ell^{\prime} h X$, semi-inclusive hadron production in deep-inelastic lepton-nucleon scattering) and of Drell-Yan (DY) process ( $h N \rightarrow \ell \bar{\ell} X$, massive lepton-pair production in hadron-nucleon collisions). Applying the TMD factorization theorems [3] allows one to express the asymmetries raising in DY and SIDIS cross sections in terms of convolutions of perturbatively calculable hard-scattering parton cross sections, hard-scale dependent TMD PDFs and (for SIDIS) parton fragmentation functions (FFs). The hard scale $Q$ in SIDIS is given by the square root of the virtuality of the photon exchanged in the DIS process and in DY by the invariant mass of the produced lepton pair. Measurement and following study of the spin (in)dependent azimuthal effects in SIDIS and Drell-Yan is a powerful method to access TMD distribution functions of the nucleon, which in past decades became a priority direction in experimental and theoretical high-energy physics, for recent reviews see e.g. Refs. [4, 5, 6].

Following general considerations (e.g. assuming single photon exchange) and standard SIDIS notations the expression for the cross section of unpolarised-hadron production in polarised-lepton SIDIS off a polarized nucleon can be written in a following model-independent way $[1,2,7]$ :

$$
\begin{aligned}
\frac{d \sigma}{d x d y d z p_{T} d p_{T} d \phi_{h} d \phi_{S}} \propto & \hat{\sigma}_{U}\left\{1+\varepsilon A_{U U}^{\cos 2 \phi_{h}} \cos 2 \phi_{h}+\sqrt{2 \varepsilon(1+\varepsilon)} A_{U U}^{\cos \phi_{h}} \cos \phi_{h}+\lambda \sqrt{2 \varepsilon(1-\varepsilon)} A_{L U}^{\sin \phi_{h}} \sin \phi_{h}\right. \\
+ & S_{L}\left[\sqrt{2 \varepsilon(1+\varepsilon)} A_{U L}^{\sin \phi_{h}} \sin \phi_{h}+\varepsilon A_{U L}^{\sin 2 \phi_{h}} \sin 2 \phi_{h}\right] \\
+ & S_{L} \lambda\left[\sqrt{1-\varepsilon^{2}} A_{L L}+\sqrt{2 \varepsilon(1-\varepsilon)} A_{L L}^{\cos \phi_{h}} \cos \phi_{h}\right] \\
+ & S_{T}\left[A_{U T}^{\sin \left(\phi_{h}-\phi_{S}\right)} \sin \left(\phi_{h}-\phi_{S}\right)+\varepsilon A_{U T}^{\sin \left(\phi_{h}+\phi_{S}\right)} \sin \left(\phi_{h}+\phi_{S}\right)+\varepsilon A_{U T}^{\sin \left(3 \phi_{h}-\phi_{S}\right)} \sin \left(3 \phi_{h}-\phi_{S}\right)\right. \\
& \left.+\sqrt{2 \varepsilon(1+\varepsilon)} A_{U T}^{\sin \phi_{S}} \sin \phi_{S}+\sqrt{2 \varepsilon(1+\varepsilon)} A_{U T}^{\sin \left(2 \phi_{h}-\phi_{S}\right)} \sin \left(2 \phi_{h}-\phi_{S}\right)\right] \\
+ & S_{T} \lambda\left[\sqrt{\left(1-\varepsilon^{2}\right)} A_{L T}^{\cos \left(\phi_{h}-\phi_{S}\right)} \cos \left(\phi_{h}-\phi_{S}\right)\right. \\
& \left.\left.+\sqrt{2 \varepsilon(1-\varepsilon)} A_{L T}^{\cos \phi_{S}} \cos \phi_{S}+\sqrt{2 \varepsilon(1-\varepsilon)} A_{L T}^{\cos \left(2 \phi_{h}-\phi_{S}\right)} \cos \left(2 \phi_{h}-\phi_{S}\right)\right]\right\}
\end{aligned}
$$

where $\hat{\sigma}_{U}=\left(F_{U U, T}+\varepsilon F_{U U, L}\right)$ while $F_{U U, T}, F_{U U, L}$ are the polarization and azimuth-independent structure functions and $\varepsilon=\left(1-y-\frac{1}{4} \gamma^{2} y^{2}\right) /\left(1-y+\frac{1}{2} y^{2}+\frac{1}{4} \gamma^{2} y^{2}\right)$ is the ratio of longitudinal and transverse photon fluxes with $\gamma=2 M x / Q$. The longitudinal and transverse components of the target polarization vector $\mathbf{S}$ are denoted as $S_{L}$ and $S_{T}$, respectively. Schematic view of the $\gamma^{*} N$

\footnotetext{
${ }^{1} \mathrm{QCD}$ generalized universality: time-reversal modified process-independence of TMD PDFs
} 
coordinate system is presented in Fig. 1. The azimuthal angles $\phi_{h}$ and $\phi_{S}$ define correspondingly the azimuthal orientation of the hadron momentum and the nucleon spin with respect to the lepton scattering plane calculated about the virtual-photon momentum direction.

Throughout this Letter the asymmetries are denoted as, $A_{X Y}^{w}$, and are defined as the amplitudes of the corresponding azimuthal modulations, $w$, divided by the spin and azimuth-independent part of the given cross section (SIDIS or DY), $\hat{\sigma}_{U}$, the effective proton polarization ${ }^{23}$ and corresponding depolarization factor ${ }^{4}$. Here, the subscript $\mathrm{X}$ is valid only for SIDIS-case and indicates the state of the lepton polarization, $\lambda$, (either U-unpolarised, or Llongitudinal), while $\mathrm{Y}$ stands for the target polarization

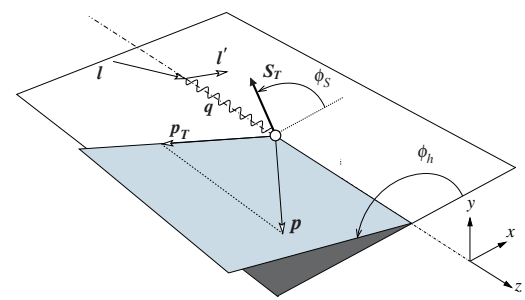

Figure 1: The SIDIS framework. (U-unpolarized, L-longitudinal or T-transverse).

In this Letter only spin-dependent effects are considered. The cross section expression from Eq. 1.1 contains three target spin independent, four (one azimuth-independent and three azimuthal) target longitudinal-spin-dependent asymmetries (LSAs) and eight transverse target-polarizationdependent azimuthal asymmetries (TSAs). Two LSAs and five TSAs depend only on nucleon polarization and are known as single spin asymmetries (SSA), while the remaining spin-dependent asymmetries entering in Eq. 1.1 depend both on nucleon and lepton polarizations and are known as double spin asymmetries (DSAs).

Within the QCD parton model approach, 2 LSAs and 4 TSAs (marked in red in Eq. 1.1) have pure twist-2 interpretations in terms of convolutions of TMD PDFs and FFs, see Table 1. The other 6 target spin-dependent SIDIS asymmetries are at sub-leading order in $Q^{-1}$ (marked in blue in Eq. 1.1), and involve a mixture of twist-2 and, induced by largely unexplored quark-gluon correlations, twist-3 parton distribution and fragmentation functions. However, by applying the widely adopted simplification known as Wandzura-Wilczek approximation (WWA) [8], these higher twist functions can be simplified to the twist-2 level $[1,2]$. Corresponding relations ${ }^{5}$ are listed in Table 2. The $A_{U L}^{\sin \left(2 \phi_{h}\right)}$ is the only azimuthal LSA arising in the

Table 1: Interpretation of LO LSAs and TSAs.

\begin{tabular}{cc}
\hline LO LSA/TSA & $P D F_{\text {twist }-2} \otimes F F_{\text {twist }-2}$ \\
\hline$A_{U L}^{\sin \left(2 \phi_{h}\right)}$ & $h_{1 L}^{\perp q} \otimes H_{1 q}^{\perp h}$ \\
\hline$A_{L L}$ & $g_{1 L}^{q} \otimes D_{1 q}^{h}$ \\
\hline$A_{U T}^{\sin \left(\phi_{h}-\phi_{S}\right)}$ & $f_{1 T}^{\perp q} \otimes D_{1 q}^{h}$ \\
\hline$A_{U T}^{\sin \left(\phi_{h}+\phi_{S}-\pi\right)}$ & $h_{1}^{q} \otimes H_{1 q}^{\perp h}$ \\
\hline$A_{U T}^{\sin \left(3 \phi_{h}-\phi_{S}\right)}$ & $h_{1 T}^{\perp q} \otimes H_{1 q}^{\perp h}$ \\
\hline$A_{L T}^{\cos \left(\phi_{h}-\phi_{S}\right)}$ & $g_{1 T}^{q} \otimes D_{1 q}^{h}$ \\
\hline
\end{tabular}
SIDIS cross section at leading order. It gives access to the convolution of so far unknown $h_{1 L}^{\perp q}$ TMD PDF, which describes transversely polarized quarks in the longitudinally polarized nucleon, and the extensively studied Collins fragmentation function, $H_{1 q}^{\perp h}[5,6]$.

The next two azimuthal LSAs, $A_{U L}^{\sin \left(\phi_{h}\right)}$ and $A_{L L}^{\cos \left(\phi_{h}\right)}$, enter the cross-section at subleading level (additional dynamic $Q^{-1}$ suppression). At the twist-3 level polarized structure functions associated

\footnotetext{
${ }^{2}$ effective polarization accounts for the dilution factor $f$ which describes the fraction of polarizable material in the target

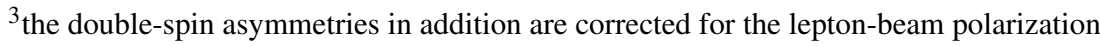

${ }^{4}$ in SIDIS the depolarization factors are the $\varepsilon$-depend coefficients standing in front of the asymmetries in Eq. 1.1

${ }^{5}$ here convolutions involving twist-3 FFs are omitted
} 
Table 2: Interpretation of sub-leading LSAs and TSAs.

\begin{tabular}{ccc}
\hline sub-leading LSA/TSA & $P D F_{\text {twist-3 }} \otimes F F_{\text {twist-2 }}$ & WWA: $P D F_{\text {twist }-2} \otimes F F_{\text {twist }-2}$ \\
\hline$A_{U L}^{\sin \left(\phi_{h}\right)}$ & $x h_{L}^{q} \otimes H_{1 q}^{\perp h}, x f_{L}^{\perp q} \otimes D_{1 q}^{h}$ & $h_{1 L}^{\perp q} \otimes H_{1 q}^{\perp h}$ \\
\hline$A_{L L}^{\cos \left(\phi_{h}\right)}$ & $x e_{L}^{q} \otimes H_{1 q}^{\perp h}, x g_{L}^{\perp q} \otimes D_{1 q}^{h}$ & $g_{1 L}^{q} \otimes D_{1 q}^{h}$ \\
\hline$A_{U T}^{\sin \left(\phi_{S}\right)}$ & $x f_{T}^{q} \otimes D_{1 q}^{h}, x h_{T}^{q} \otimes H_{1 q}^{\perp h}, x h_{T}^{\perp q} \otimes H_{1 q}^{\perp h}$ & $f_{1 T}^{\perp q} \otimes D_{1 q}^{h}, h_{1}^{q} \otimes H_{1 q}^{\perp h}$ \\
\hline$A_{U T}^{\sin \left(2 \phi_{h}-\phi_{S}\right)}$ & $x f_{T}^{\perp q} \otimes D_{1 q}^{h}, x h_{T}^{q} \otimes H_{1 q}^{\perp h}, x h_{T}^{\perp q} \otimes H_{1 q}^{\perp h}$ & $f_{1 T}^{\perp q} \otimes D_{1 q}^{h}, h_{1}^{q} \otimes H_{1 q}^{\perp h}$ \\
\hline$A_{L T}^{\cos \left(\phi_{S}\right)}$ & $x g_{T}^{q} \otimes D_{1 q}^{h}, x e_{T}^{q} \otimes H_{1 q}^{\perp h}, x e_{T}^{\perp q} \otimes H_{1 q}^{\perp h}$ & $g_{1 T}^{q} \otimes D_{1 q}^{h}$ \\
\hline$A_{L T}^{\cos \left(2 \phi_{h}-\phi_{S}\right)}$ & $x g_{T}^{\perp q} \otimes D_{1 q}^{h}, x e_{T}^{q} \otimes H_{1 q}^{\perp h}, x e_{T}^{\perp q} \otimes H_{1 q}^{\perp h}$ & $g_{1 T}^{q} \otimes D_{1 q}^{h}$ \\
\hline
\end{tabular}

to these two longitudinal SSAs include four individual contributions given by couplings of specific twist-2 and twist-3 PDFs and FFs [2]. Keeping only the contributions involving twist-2 FFs, the $A_{U L}^{\sin \left(\phi_{h}\right)}$ asymmetry is described by a combination of two such couplings: $x h_{L}^{q} \otimes H_{1 q}^{\perp h}$ and $x f_{L}^{\perp q} \otimes$ $D_{1 q}^{h}$, where $D_{1 q}^{h}$ is the ordinary fragmentation function. The $x f_{L}^{\perp q}$ is a T-odd TMD PDF that can be interpreted as the twist-3 analog of the Sivers function, while $x h_{L}^{q}$ is a chiral-odd PDF which enters in convolution with Collins FF. The contributions coming from both twist-3 PDFs have been recently calculated in two different spectator-diquark models [9].

The $A_{L L}^{\cos \left(\phi_{h}\right)}$ LSA, is related to the $x e_{L}^{q} \otimes H_{1 q}^{\perp h}$ and $x g_{L}^{\perp q} \otimes D_{1 q}^{h}$ couplings which within WWA simplify to $g_{1 L}^{q} \otimes D_{1 q}^{h}$, where $g_{1 L}^{q}$ is the TMD helicity PDF. This allows to link this asymmetry to another longitudinal DSA, the azimuth-independent $A_{L L}$ LO double-spin asymmetry. The $A_{L L}$ is related to the same $g_{1 L}^{q} \otimes D_{1 q}^{h}$ convolution and serves as a clean source to access TMD helicity PDF. In particular, as it was suggested in [10], the behaviour of the $A_{L L}$ asymmetry as a function of $p_{T}$ can be used to disentangle different scenarios of transverse momentum dependence of the $g_{1 L}^{q}$ TMD PDF.

Out of eight TSAs that appear in SIDIS cross-section in Eq. 1.1, four are LO asymmetries induced by twist-2 TMD PDFs, see Table 1. The first two LO SSAs, "Sivers" $\left(A_{U T}^{\sin \left(\phi_{h}-\phi_{S}\right)}\right)$ and "Collins" $\left(A_{U T}^{\sin \left(\phi_{h}+\phi_{S}\right)}\right)$ effects, are the most studied ones [4, 5, 6]. Corresponding structure functions are given as convolutions of Sivers $\left(f_{1 T}^{\perp}\right)$ TMD PDF with $D_{1 q}^{h}$ FF and transversity $\left(h_{1}\right)$ TMD PDF with the Collins FF, respectively. The other two LO terms give access to another pair of twist-two TMD PDFs: $A_{U T}^{\sin \left(3 \phi_{h}-\phi_{S}\right)}$ SSA is related to pretzelosity $\left(h_{1 T}^{\perp}\right)$ TMD PDF [11, 12] and Collins FF, while $A_{L T}^{\cos \left(\phi_{h}-\phi_{S}\right)}$ double-spin asymmetry gives access to the worm-gear $\left(g_{1 T}\right)$ function convoluted with $D_{1 q}^{h}$ ordinary FF [13]. Remaining four TSAs are subleading effects related to twist-3 PDFs and FFs [2], which can be simplified to the twist-2 level using WWA (see Table 2). It is interesting to note that within WWA the $A_{U T}^{\sin \left(\phi_{S}\right)}$ SSA can be interpreted as a combination of Sivers and Collins constituents.

Among the TSAs, an important role is played by the Sivers asymmetry and related twist-2 Sivers function $\left(f_{1 T}^{\perp}\right)$ [14]. Similarly to the SIDIS case the nucleon TMD PDFs induce analogous azimuthal effects in the cross-section of Drell-Yan process. A peculiar feature of the Sivers TMD PDF predicted in Refs. [15, 16, 17] is that along with the second time-reversal odd TMD PDF, Boer-Mulders function $\left(h_{1}^{\perp}\right.$ ), it contributes with opposite sign to SIDIS and DY (or W/Z-boson production), which is considered to be an essential prediction of TMD framework of QCD and a 
major challenge in hadron physics. In contrast to the Sivers and Boer-Mulders functions, other functions e.g. transversity and pretzelosity TMD PDFs are predicted to be genuinely universal, which means they do not change sign between SIDIS and DY [3]. This is yet another fundamental QCD prediction to be explored. The COMPASS experiment at CERN [18, 19] has the unique capability to explore the transverse-spin structure of the nucleon in a similar kinematic region by two alternative experimental approaches, i.e. SIDIS and pion induced DY, using mostly the same setup and transversely polarized targets.

When the polarizations of the produced leptons are summed over, the general expression for the cross section of pion induced DY lepton-pair production off a transversely polarized nucleon comprises one polar asymmetry, two unpolarized and five transverse-spin-dependent azimuthal asymmetries. Adopting general notations and conventions of Refs. [20, 19], the corresponding model-independent differential cross section can be written as follows:

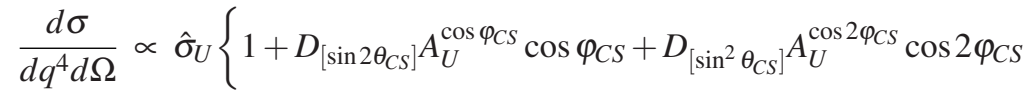

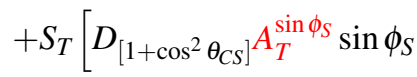

$$
\begin{aligned}
& +D_{\left[\sin 2 \theta_{C S}\right]}\left(A_{T}^{\sin \left(\varphi_{C S}-\phi_{S}\right)} \sin \left(\varphi_{C S}-\phi_{S}\right)+A_{T}^{\sin \left(\varphi_{C S}+\phi_{S}\right)} \sin \left(\varphi_{C S}+\phi_{S}\right)\right) \\
& \left.\left.+D_{\left[\sin ^{2} \theta_{C S}\right]}\left(A_{T}^{\sin \left(2 \varphi_{C S}-\phi_{S}\right)} \sin \left(2 \varphi_{C S}-\phi_{S}\right)+A_{T}^{\sin \left(2 \varphi_{C S}+\phi_{S}\right)} \sin \left(2 \varphi_{C S}+\phi_{S}\right)\right)\right]\right\}
\end{aligned}
$$

Here, $\hat{\sigma}_{U}=\left(F_{U}^{1}+F_{U}^{2}\right)\left(1+\lambda \cos ^{2} \theta_{C S}\right)$ where $F_{U}^{1}, F_{U}^{2}$ are the polarization and azimuth-independent structure functions and $\lambda$ is the polar angle asymmetry, given as $\lambda=\left(F_{U}^{1}-F_{U}^{2}\right) /\left(F_{U}^{1}+F_{U}^{2}\right)$. The virtual-photon depolarization factors are defined as, $D_{\left[f\left(\theta_{C S}\right)\right]}=f\left(\theta_{C S}\right) /\left(1+\lambda \cos ^{2} \theta_{C S}\right)$ with $f\left(\theta_{C S}\right)$ being equal either to $\sin 2 \theta_{C S}$, or $\sin ^{2} \theta_{C S}$, or to $1+\cos ^{2} \theta_{C S}$ depending on the TSA. The angles $\varphi_{C S}, \theta_{C S}$ and $\Omega$, the solid angle of the lepton, are defined in the Collins-Soper frame following the considerations of Refs. [20, 19], and $\phi_{S}$ is the azimuthal angle of the direction of the nucleon polarization in the target rest frame, see Fig. 2. Three out of five TSAs enter the cross-section at leading order of perturbative QCD and can be described by contributions from only twist-2 TMD PDFs. The TSA $A_{T}^{\sin \phi_{S}}$ is related to the nucleon Sivers TMD PDF $\left(f_{1 T}^{\perp}\right)$ convoluted with the unpolarized pion TMD PDF $\left(f_{1, \pi}\right)$. Here, following similar SIDIS conventions, twist- 3 contribution to the Sivers TSA is neglected (the $F_{U T}^{2}$ structure function is assumed to be zero [19]). Analogously, within the twist-2 approximation of LO pQCD, $F_{U}^{2}=0$, which leads to $\lambda=1$. The other two twist-2 TSAs, $A_{T}^{\sin \left(2 \varphi_{C S}-\phi_{S}\right)}$ and $A_{T}^{\sin \left(2 \varphi_{C S}+\phi_{S}\right)}$, are related to convolutions of the Boer-Mulders TMD PDF $\left(h_{1, \pi}^{\perp}\right)$ of the
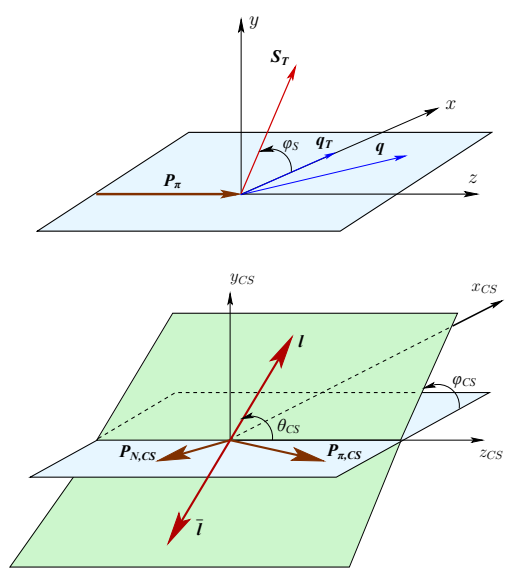

Figure 2: The target rest (top) and the Collins-Soper frames (bottom). pion with the nucleon TMD PDF transversity $\left(h_{1}\right)$ and pretzelosity $\left(h_{1 T}^{\perp}\right)$, respectively [20, 2]. Remaining two TSAs, namely $A_{T}^{\sin \left(\varphi_{C S}-\phi_{S}\right)}$ and $A_{T}^{\sin \left(\varphi_{C S}+\phi_{S}\right)}$ are subleading-twist structures and are expected to vanish at LO. 
During past two decades HERMES, CLAS and COMPASS performed series of SIDIS measurements addressing LSAs [21, 22, 23, 24] and TSAs [25, 26, 27, 28, 29]. Non-zero quark Sivers TMD PDFs have been extracted from HERMES [26] and COMPASS [25, 27, 30] data using both collinear [31] and TMD $Q^{2}$-evolution approaches [32, 33, 34]. The first measurement of the Sivers effect in $W$ and $Z$-boson production in collisions of transversely polarized protons at RHIC was reported by the STAR collaboration [35]. The hard scales of these measurements are by factor of 10 larger compared to the ones explored in fixed-target experiments where $Q$ ranges approximately between $1 \mathrm{GeV} / c$ and $9 \mathrm{GeV} / c$. In the commonly accessible range of the Bjorken- $x$ variable, the Sivers TSA at HERMES was found to be somewhat larger compared to that measured at COMPASS. Taking into account that in this range the hard scale at COMPASS is as much as two to three times larger compared to that of HERMES, this observation may indicate the influence of TMD evolution effects. Hence it is not excluded that TMD evolution effects may be sizable when describing the STAR results using Sivers TMD PDFs extracted from fixed-target SIDIS results. In order to further test this conjecture and provide a valuable input for testing the effect of TMD evolution, COMPASS recently performed the first multi-differential analysis of the TSAs extracting them from SIDIS data at four different hard scales [36]. These $Q^{2}$ regions were chosen to match the four regions of the di-muon mass used in the ongoing analyses of the COMPASS Drell-Yan measurements, which allows for a direct comparison of the nucleon TMD PDFs extracted from these two alternative measurements. Finally, COMPASS recently provided the missing input for this comparison publishing the results for first ever measurements of three twist-2 Drell-Yan TSAs done for the invariant mass range between $4.3 \mathrm{GeV} / c^{2}$ and $8.5 \mathrm{GeV} / c^{2}[37,38]$.

In this Letter recent COMPASS SIDIS and Drell-Yan results for target-spin-dependent asymmetries will be presented. This includes: preliminary results obtained for proton SIDIS LSAs, review of published SIDIS results for TSAs extracted at four hard scales of the Drell-Yan process at COMPASS and brief summary of recently published proton transverse-spin-dependent asymmetries in the pion-induced Drell-Yan process.

\section{Data analysis}

COMPASS results for proton SIDIS asymmetries presented in this Letter are based on data collected using a naturally polarized $\mu^{+}$-beam (from the CERN SPS - M2 beamline) of nominal energy of $160 \mathrm{GeV} / c(200 \mathrm{GeV} / \mathrm{c}$ in 2011) impinging on a three celled $(30 \mathrm{~cm}, 60 \mathrm{~cm}, 30 \mathrm{~cm})$ proton $\left(\mathrm{NH}_{3}\right)$ target, polarized longitudinally (in 2007 and 2011) or transversely (in 2010). The data of each year were scrutinized separately by various stability and quality tests.

In order to ensure the DIS-regime the negative square of the virtual photon's four momentum, $Q^{2}$, is required to fulfill the condition $Q^{2}>1(\mathrm{GeV} / \mathrm{c})^{2}$. Contributions from exclusive nucleon resonance production are cut by the restriction on the invariant mass of the $\gamma^{*} N$ system, $W>5 \mathrm{GeV} / \mathrm{c}^{2}\left(W>\sqrt{10} \mathrm{GeV} / \mathrm{c}^{2}\right.$ in $2010 \mathrm{TSA}$-analysis $)$. The Bjorken scaling variable is limited to $0.0025<x<0.7$. Furthermore, the fractional energy of the virtual photon is limited to $0.1<y<0.9$. Here, the upper $y$ cut rejects events, where radiative corrections become sizable, while the lower $y$ cut removes the elastic tail and rejects events, where halo or background muons are misidentified as scattered muons. To ensure a good resolution of the measured angle $\phi_{h}$, the transverse component of the momentum of the hadron is required to satisfy the criteria 
$p_{T}>0.1 \mathrm{GeV} / \mathrm{c}$. The fractional energy of each hadron is limited to $0.1<z<1.0$. The asymmetries were extracted for three $z$ intervals: $z>0.1, z>0.2$ and $0.1<z<0.2$.

After all cuts in $z>0.1$ range there are in total about $10^{8}, 0.8 \cdot 10^{8}$ and $1.6 \cdot 10^{8}$ charged hadrons for 2007, 2011 and 2010, respectively. The different beam energies in 2007 and 2011 explain some relatively small deviations in $x, Q^{2}, y$, and $W$, which still allowed to merge the two samples and to study the LSAs for combined 2007-2011 data set.

The SIDIS asymmetries are extracted in bins of $x, z, p_{T}$ and $W$ using standard COMPASS extraction methods. In addition to these one-dimensional dependences the TSAs are extracted for the first time using two-dimensional representations in $\left(Q^{2}, x\right),\left(Q^{2}, z\right)$, and $\left(Q^{2}, p_{T}\right)$. The twodimensional extractions are done using four $Q^{2}$-ranges close to those selected for the analysis of the DY data. The $Q^{2}$-ranges are defined by the following bin limits applied to $Q: 1,2,2.5,4,9$ (in units of $\mathrm{GeV} / c$ ). More details on SIDIS analyses can be found in Refs. [24, 36].

The DY analysis presented in this Letter is based on Drell-Yan data collected by COMPASS in the year 2015 using essentially the same spectrometer as it was used during SIDIS data taking in previous years [19]. For this measurement, the $190 \mathrm{GeV} / c \pi^{-}$beam with an average intensity of $0.6 \times 10^{8} \mathrm{~s}^{-1}$ was scattered off the COMPASS transversely polarized $\mathrm{NH}_{3}$ target. The polarized target consisted of two longitudinally aligned cylindrical cells of $55 \mathrm{~cm}$ length and $4 \mathrm{~cm}$ in diameter, separated by a $20 \mathrm{~cm}$ gap. In the data analysis, the selection of events requires a production vertex located within the polarized-target volume, with one incoming pion beam track and at least two oppositely charged outgoing particles that are consistent with the muon hypothesis, i.e. they crossed at least 30 radiation lengths of material along the spectrometer. The mass range used in this analysis is defined by the requirement $4.3 \mathrm{GeV} / c^{2}<M_{\mu \mu}<8.5 \mathrm{GeV} / c^{2}$, where the upper limit avoids the contribution of $\Upsilon$-resonances. In this range, the overall background contribution is estimated to be below $4 \%$. The dimuon transverse momentum $q_{T}$ is required to be above $0.4 \mathrm{GeV} / c$ in order to obtain sufficient resolution in angular variables. After all selection criteria about $35 \times 10^{3}$ dimuons remain for the analysis. The asymmetries are evaluated in kinematic bins of $x_{N}, x_{\pi}, x_{F}$ or $q_{T}$, while always integrating over all the other variables.

A set of systematic studies has been performed to test the stability of obtained SIDIS and DY results over the given data-taking year and to identify possible systematic biases via study of various false asymmetries. The studies were done in a similar way as described in Refs. [27, 28]. The results of false-asymmetry tests coupled with the outcome of periods compatibility test and results of the monitoring of the mean values of the asymmetries on period-by-period basis, have not given any critical indications for any of the data-taking periods. Apart from these studies, the SIDIS LSAs (TSAs) were corrected for admixtures due to non-vanishing transverse (longitudinal) $\gamma^{*} N$ polarization components $[24,7]$. The final systematic point-to-point uncertainties are indicated in the plots by color bands. In the SIDIS plots the abscissa positions of the points for negative hadrons are slightly shifted to the right for better visibility.

\section{SIDIS results with longitudinally polarized target}

The six LSAs that are extracted from COMPASS SIDIS data of 2007 and 2011 in this analysis are shown in Figs. 3-4. The LSAs, $A_{L L}, A_{U L}^{\sin \left(\phi_{h}\right)}, A_{U L}^{\sin \left(2 \phi_{h}\right)}$ and $A_{U L}^{\cos \left(\phi_{h}\right)}$ extracted for two different $z$-ranges $(0.1<z<0.2$ and $z>0.2)$ are shown in Fig. 3 as a function of the variables $x, z$ and 

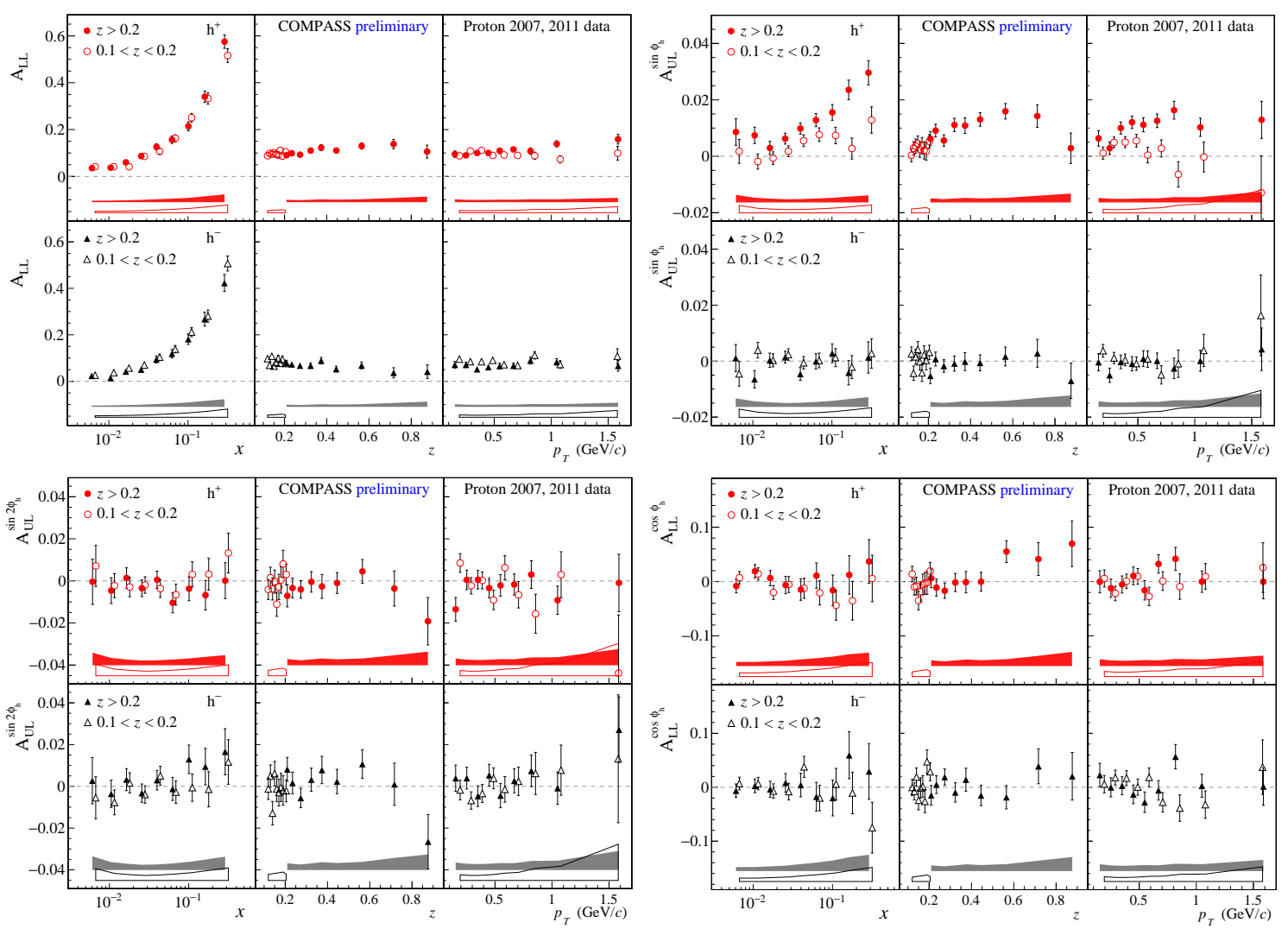

Figure 3: Kinematic dependences of $A_{L L}, A_{U L}^{\sin \left(\phi_{h}\right)}, A_{U L}^{\sin \left(2 \phi_{h}\right)}$ and $A_{U L}^{\cos \left(\phi_{h}\right)}$ LSAs.

$p_{T}$. In the Fig. 4 the LSAs obtained by COMPASS ( $x$-dependence only) are superimposed with available theoretical predictions $[10,39,40]$ and the experimental results obtained previously by the HERMES collaboration [21].

The azimuth-independent $A_{L L}$ asymmetry shown in Fig. 3 (top left panel) as expected exhibits strong $x$-dependence well described by models, see Fig. 4. At the same time the $z$ and $p_{T}$ dependences of the LSA appear to be rather flat. As suggested in Ref. [10], flat behaviour of the $A_{L L}$ as a function of $p_{T}$ could point to the similarity of the average quark transverse motion inside unpolarized and longitudinally polarized nucleons.

Apart from the well-known large effect for $A_{L L}$-asymmetry, all other LSAs according to various model calculations are predicted to be small both at COMPASS and HERMES kinematics, reaching in absolute maximal values of $\simeq 0.05$ in the region of large $x(x>0.1)$ [10, 39, 40]. At COMPASS the only azimuthal LSA which exhibits clear non-zero behaviour is the $A_{U L}^{\sin \left(\phi_{h}\right)}$ subleading amplitude. Averaged $A_{U L}^{\sin \left(\phi_{h}\right)}$ LSA for positive hadrons is found to be about nine standard deviations above zero and obtained results for $A_{U L}^{\sin \left(\phi_{h}\right)}$ appear to be in qualitative agreement with theoretical predictions from Ref. [9] done for COMPASS kinematics. This measurement for positive hadrons confirms with much higher precision the previous non-zero observations made by the HERMES collaboration [21] (see Fig. 4 top right panel). Nevertheless, making direct comparison with HERMES results is not worthwhile. The asymmetry exhibits strong $z$-dependence and is sensitive to $Q$ because of its subleading nature $\left(Q^{-1}\right.$ suppression), while two experiments have 

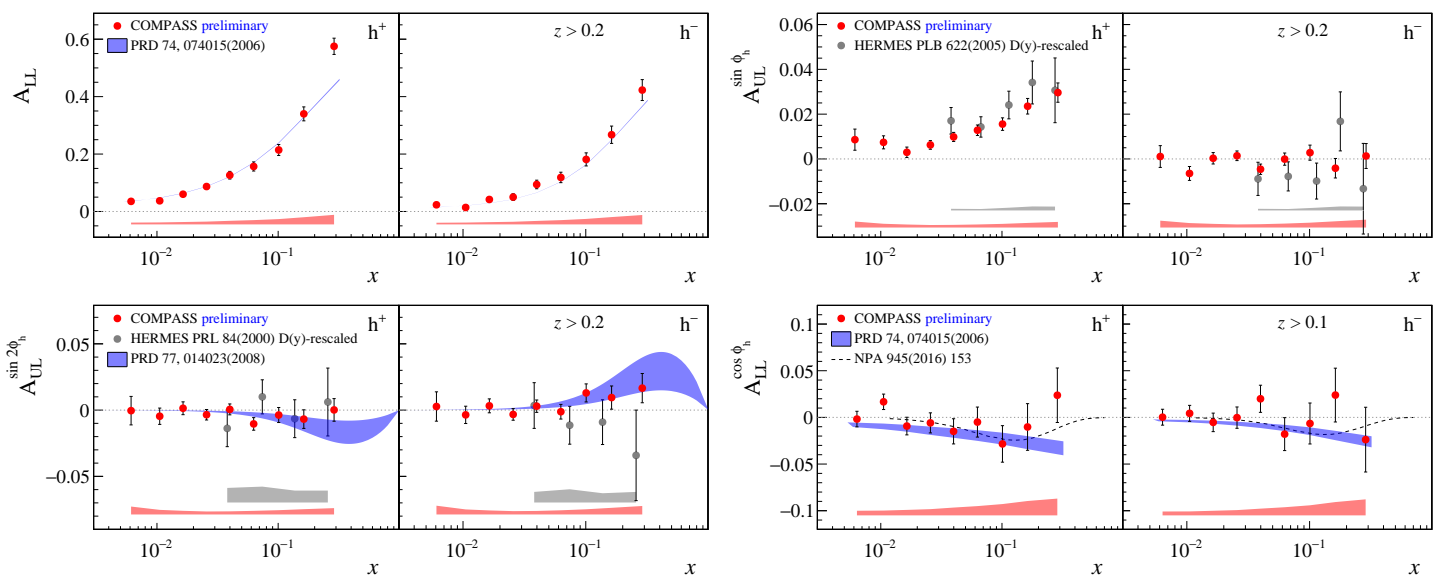

Figure 4: The $A_{L L}, A_{U L}^{\sin \left(\phi_{h}\right)}, A_{U L}^{\sin \left(2 \phi_{h}\right)}$ and $A_{U L}^{\cos \left(\phi_{h}\right)}$ LSAs are shown together with HERMES results (re-scaled by the corresponding $D(y)$ factors) and available model predictions [10, 39, 40].

different coverage for both these variables.

The $A_{U L}^{\sin \left(2 \phi_{h}\right)}$ term is the only azimuthal LSA arising in the SIDIS cross section at leading order and thus doesn't suffer from $Q^{-1}$ suppression-factor. Nevertheless, the asymmetry comes with kinematic pre-factor which effectively leads to relative $\sim\left|\overrightarrow{p_{T}}\right|$-suppression of the effect. At large $x$ there are some hints for a small and statistically unclear (about 1.5-2 standard deviations away from zero) "mirror-symmetric" effect for oppositely charged hadrons. This agrees with model calculations [39] predicting small asymmetry with characteristic mirror-symmetric "Collins-like" behaviour induced by the presence of the $H_{1 q}^{\perp h}$ Collins FF. Corresponding theoretical curves are presented for comparison in Fig 4 together with HERMES experimental points [21].

Finally, the $A_{L L}^{\cos \left(\phi_{h}\right)}$ longitudinal DSA appears to be small with no clear trend observed due to relatively large statistical uncertainties, see Fig. 3. Existing model calculations available for this asymmetry, predict small (less than 4\%) effect, which is in agreement with COMPASS results as can be seen in Fig. 4 (bottom left panel).

\section{SIDIS results with transversely polarized target}

The eight TSAs that are extracted from COMPASS SIDIS data in this analysis are shown in Fig. 5 in the four above defined $Q^{2}$-ranges, after averaging over all other kinematic dependences. In particular, the Sivers TSA is determined with good statistical accuracy in all four $Q^{2}$-ranges. For positive hadrons its amplitude is clearly positive in all four $Q^{2}$-ranges, whereas for negative hadrons it is compatible with zero in the lowest $Q^{2}$-range and becomes significantly positive in the other three. The Collins asymmetry has a significant signal in all four $Q^{2}$-ranges with opposite sign for positive and negative hadrons, which is attributed to the peculiarities of Collins FF [28]. The pretzelosity asymmetry in SIDIS is found to be compatible with zero, which can be related to the kinematic suppression-factors entering in the corresponding structure function and to the smallness of the PDF itself $[2,11,12]$. The $A_{L T}^{\cos \left(\phi_{h}-\phi_{S}\right)}$ LO DSA exhibits significant non-zero signal in the last $Q^{2}$ bin, which is in agreement with theoretical expectations [13]. Among subleading effects 


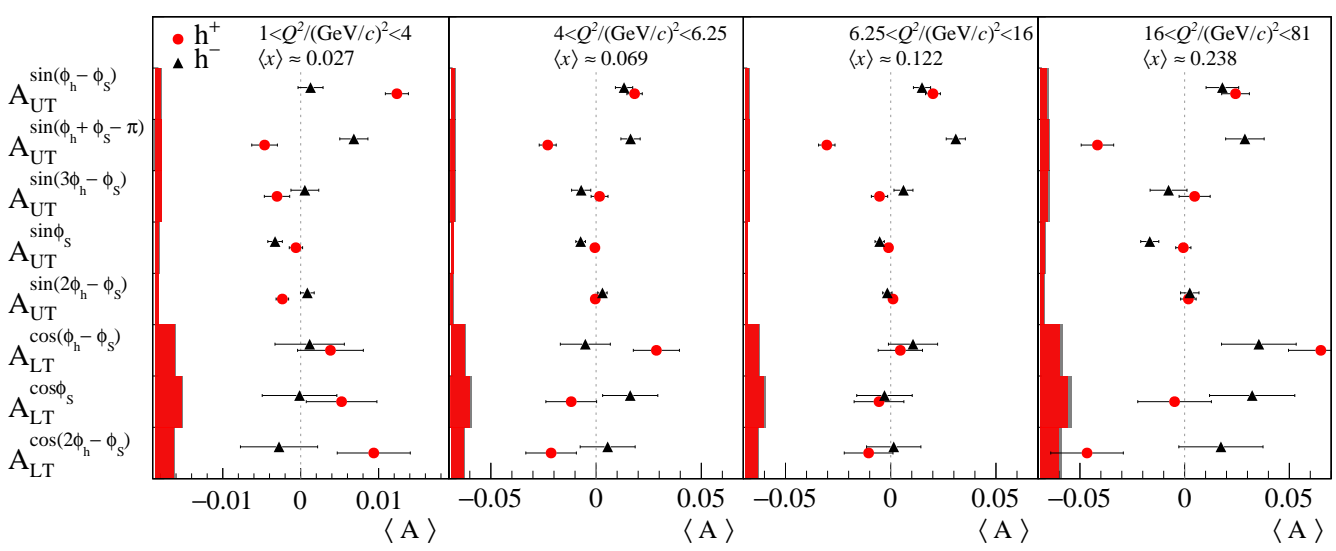

Figure 5: Mean TSAs in the four $Q^{2}$-ranges. For each $Q^{2}$-range also the average $x$-values are given.

the only non-zero asymmetry is the $A_{U T}^{\sin \left(\phi_{S}\right)}$ TSA which within WWA can be related to Sivers and Collins effects.

In the left panel of Fig. 6, the Sivers TSAs $A_{U T}^{\sin \left(\phi_{h}-\phi_{S}\right)}$ for positive and negative hadrons are shown as a function of $x, z$ and $p_{T}$ in the four above selected $Q^{2}$-ranges. For positive hadrons, a positive Sivers TSA is observed in the whole $x$-interval and in all four $Q^{2}$-ranges (first column). The Sivers asymmetry as a function of $x$ appears to increase up to $x \simeq 0.2$ in each of the $Q^{2}$ ranges, followed by a possible decrease at large $x$. The second and third columns indicate an approximately linear dependence at low $z$ and $p_{T}$ values, which is in agreement with the existing phenomenological parameterizations of the Sivers effect [31]. For negative hadrons, the Sivers TSA is sizeably smaller and less prominent. At intermediate $z(0.3 \div 0.6)$ and low $Q^{2}$ (first row) it appears to be negative. For larger values of $Q^{2}$, the Sivers TSA for negative hadrons tends to grow and becomes positive. In the left panel of Fig. 6 the $Q^{2}$-dependence of the Sivers asymmetry is shown for positive and negative hadrons in five selected bins of $\mathrm{x}$. The figure also shows the predictions from collinear (DGLAP) and TMD-evolution, which are based on the best fit [32] of all published HERMES [26] and COMPASS [25, 27] measurements. A comparison of the points from the same $x$-bins but different $Q^{2}$-ranges shows no clear $Q^{2}$-dependence of the Sivers TSAs within statistical accuracy. Also, the comparison of fits (not shown in the figure) performed with a linear decreasing function or a constant does not yield a statistically significant conclusion, although there may be a slight preference to the former dependence for positive hadrons. For negative hadrons no clear trend is observed.

Available descriptions of the Sivers TSAs, which are based on parameterizations of the unpolarised and polarised TMDs, are driven mostly by the one-dimensional data at low $x$ and low $Q^{2}$ from HERMES and COMPASS, so that present phenomenological studies of $Q^{2}$-evolution are based on fits using the results of two separate experiments. Present models predict for increasing $Q^{2}$ a slight increase of the Sivers TSAs for DGLAP and a decrease for TMD evolution. Based on the fits of one-dimensional data, various TMD-evolution models predict different sizes for the DY Sivers TSA in the high mass range, with values between 0.04 to 0.15 [32, 33, 34]. Better constraints on $Q^{2}$-evolution models of TMDs can be expected only from data that are simultaneously differential in $x$ and $Q^{2}$, as the data presented in this Letter. 

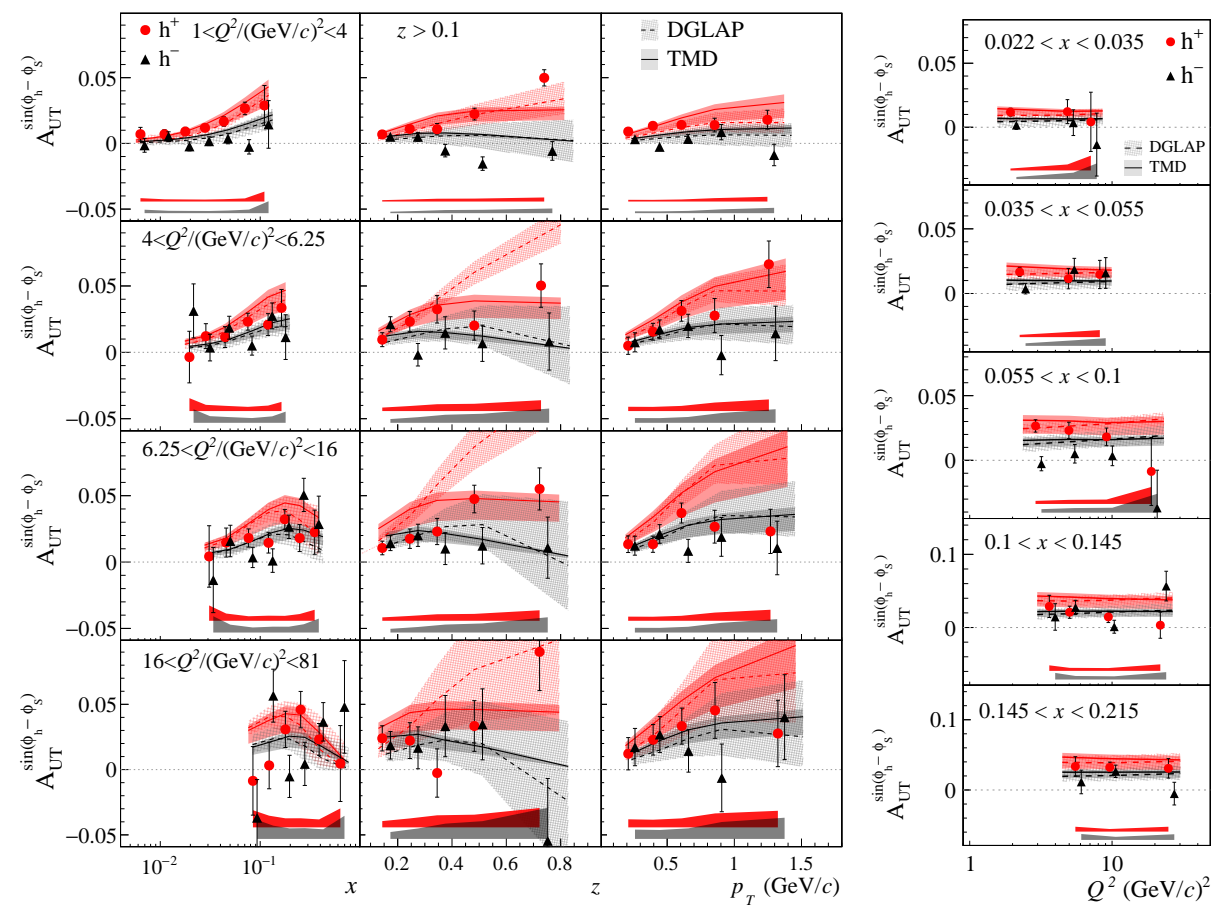

Figure 6: Left panel: Sivers asymmetry for $z>0.1$ in the four $Q^{2}$-ranges as a function of $x, z$ and $p_{T}$. Right panel: the $Q^{2}$-dependence of the Sivers asymmetry in five selected bins of $x$.
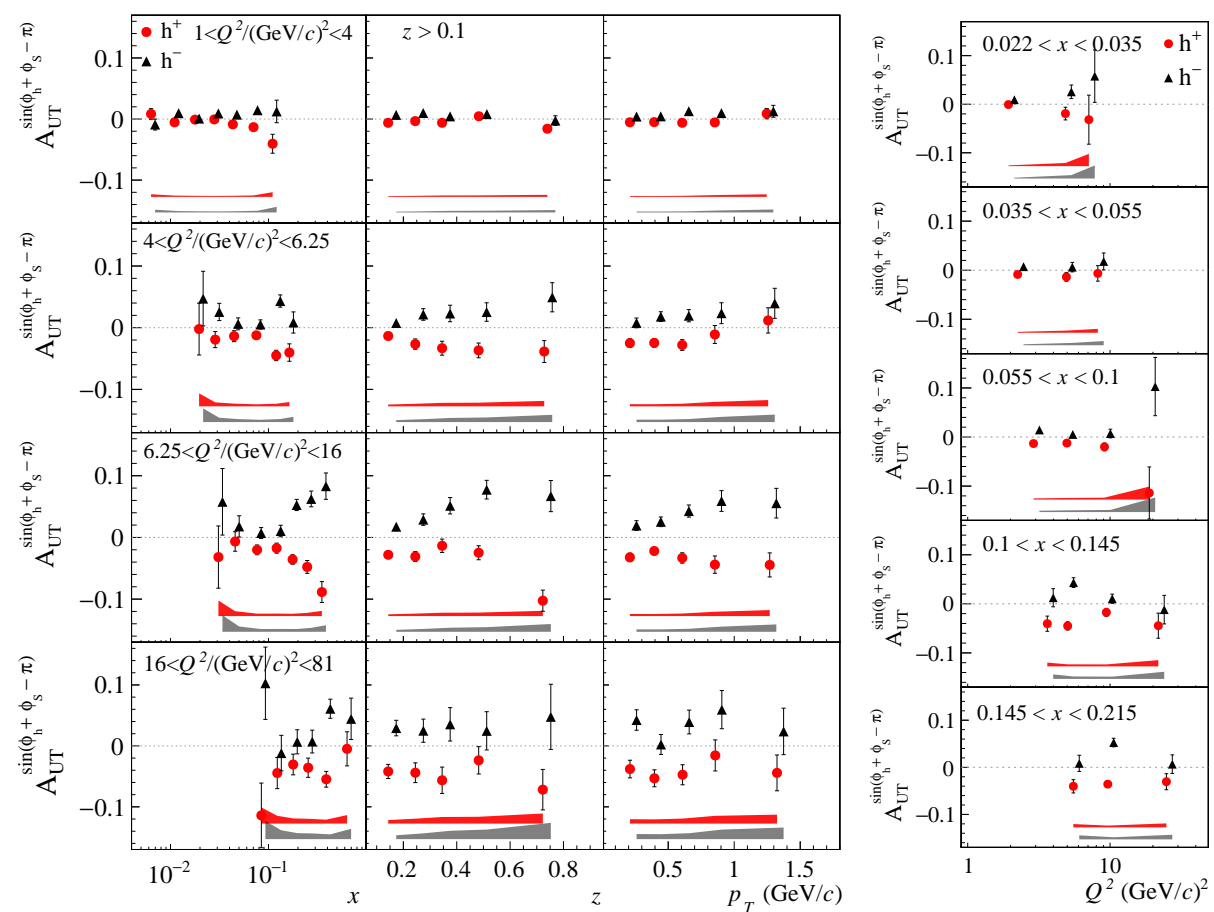

Figure 7: Left panel: Collins asymmetry for $z>0.1$ in the four $Q^{2}$-ranges as a function of $x, z$ and $p_{T}$. Right panel: the $Q^{2}$-dependence of the Collins asymmetry in five selected bins of $x$. 


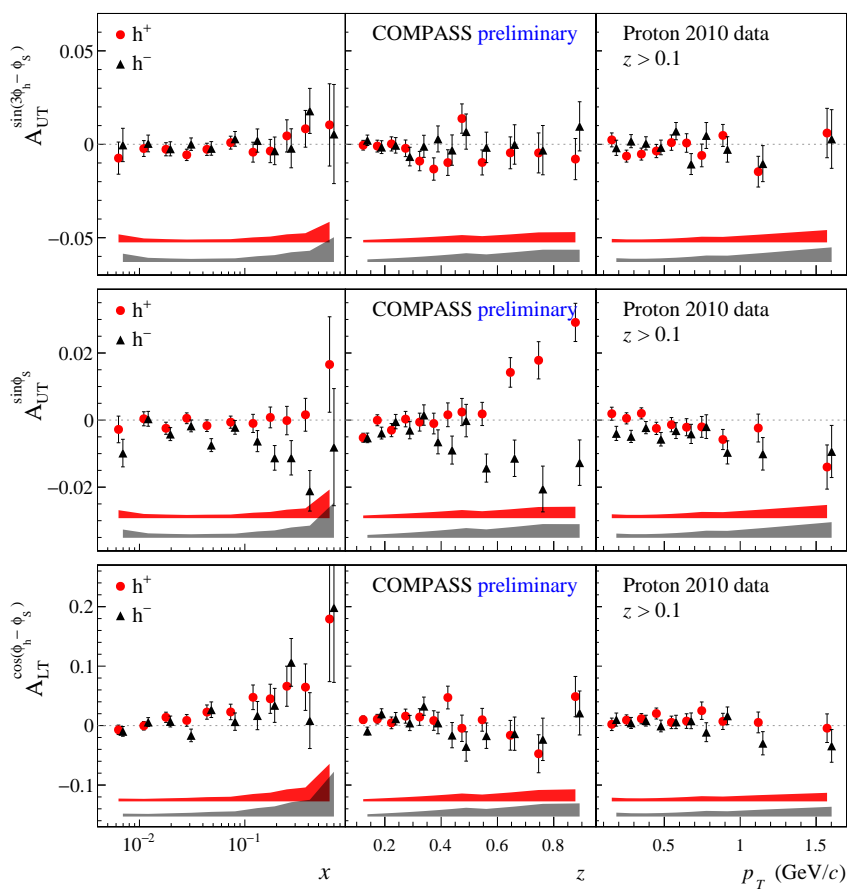

Figure 8: From top to bottom: one-dimensional representations of the $A_{U T}^{\sin \left(3 \phi_{h}-\phi_{S}\right)}, A_{L T}^{\cos \left(\phi_{h}-\phi_{S}\right)}$ and $A_{U T}^{\sin \left(\phi_{S}\right)}$ asymmetries for $z>0.1$ as a function of $x, z$ and $p_{T}$, for positive and negative hadrons.

In Fig. 7, the Collins TSAs $A_{U T}^{\sin \left(\phi_{h}+\phi_{S}-\pi\right)}$ for positive and negative hadrons are shown in twodimensional $\left(Q^{2}, x\right),\left(Q^{2}, z\right)$, and $\left(Q^{2}, p_{T}\right)$ (left panel) and $\left(x, Q^{2}\right)$ (right panel) representations. A negative (positive) Collins TSA is observed for positive (negative) hadrons in the whole $x$-interval and in all four $Q^{2}$-ranges (first column). The characteristic mirror-symmetric behaviour for oppositely charged hadrons is induced by the known peculiarities of the Collins FF [28]. The Collins asymmetry as a function of $x$ has a decreasing trend up to $x \simeq 0.1$, which is then followed by a strong increase. The dependence on $z$ and $p_{T}$ changes between different $Q^{2}$-ranges varying from slight increase to a constant behaviour. Within statistical accuracy the asymmetry doesn't exhibit any clear $Q^{2}$-dependence.

In Fig. 8, one-dimensional $x, z$ and $p_{T}$ representations of the $A_{U T}^{\sin \left(3 \phi_{h}-\phi_{S}\right)}, A_{L T}^{\cos \left(\phi_{h}-\phi_{S}\right)}$ and $A_{U T}^{\sin \left(\phi_{S}\right)}$ asymmetries, for positive and negative hadrons are shown. The $A_{U T}^{\sin \left(3 \phi_{h}-\phi_{S}\right)}$ asymmetry (top panel) appears to be small (the absolute value stays below 0.02) and compatible with zero over the full kinematical range. This is in agreement with available phenomenological calculations [11,12].

Beyond Collins and Sivers effects the only single-spin asymmetry which exhibits a non-zero behaviour is the subleading-twist $A_{U T}^{\sin \left(\phi_{S}\right)}$ TSA (middle panel). The asymmetry is small in general (the absolute value stays below 0.03), but in the region of large $z$ it is found to be above zero by more than three standard deviations of the total experimental accuracy both for positive and negative hadrons. For negative hadrons the effect is observed to be negative in the whole $x$-interval. In the bottom panel of Fig. 8, the only LO DSA $A_{L T}^{\cos \left(\phi_{h}-\phi_{S}\right)}$ for positive and negative hadrons is shown. The asymmetry is related to the worm-gear $\left(g_{1 T}\right)$ twist-2 TMD PDF, which can be linked (through Lorentz invariance relation) to twist-3 $g_{2}(x) \mathrm{PDF}$, which in its turn can be linked to twist-2 
helicity PDF $g_{1}(x)$ using WWA [13]. For positive hadrons, a positive $A_{L T}^{\cos \left(\phi_{h}-\phi_{S}\right)}$ TSA is observed in the large $x$-interval $(x>0.01)$ with an increase up to $\approx 0.1$. For negative hadrons the effect is less prominent due to larger statistical uncertainties. Observed behaviour of the effect is well in agreement with the available model calculations [13].

\section{Drell-Yan results with transversely polarized target}

All five Drell-Yan TSAs (twist-2 TSAs, $A_{T}^{\sin \phi_{S}}, A_{T}^{\sin \left(2 \varphi_{C S}-\phi_{S}\right)}$ and $A_{T}^{\sin \left(2 \varphi_{C S}+\phi_{S}\right)}$ and subleadingtwist TSAs, $A_{T}^{\sin \left(\varphi_{C S}-\phi_{S}\right)}$ and $\left.A_{T}^{\sin \left(\varphi_{C S}+\phi_{S}\right)}\right)$ are shown in Fig. 9 (left panel) as a function of the variables $x_{N}, x_{\pi}, x_{F}, q_{T}$ and $M_{\mu \mu}$. Due to relatively large statistical uncertainties, no clear trend is observed for any of the TSAs. The right panel in Fig. 9 shows the results for the five extracted TSAs integrated over the entire kinematic range. The average Sivers asymmetry $A_{T}^{\sin \phi_{S}}=$

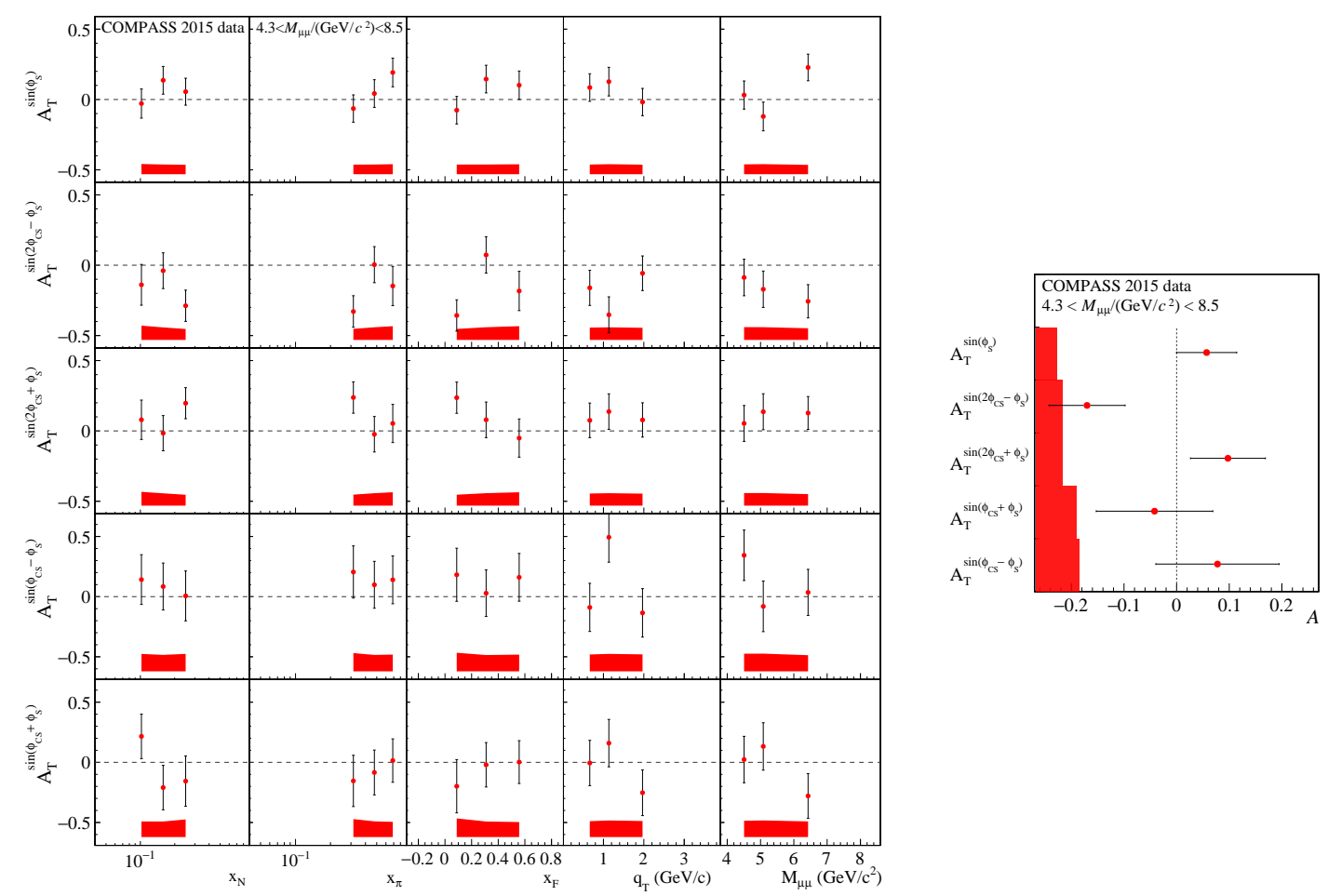

Figure 9: Left panel: Extracted Drell-Yan TSAs. Right panel: Mean Drell-Yan TSAs.

$0.060 \pm 0.057$ (stat.) \pm 0.040 (sys.) is found to be above zero at about one standard deviation of the total uncertainty. In Fig. 10, it is compared with recent theoretical predictions (with and without sign-change hypothesis) from Refs. [41, 34, 33] that are based on standard DGLAP and two different TMD evolution approaches. Note that the kinematic constraints used in Refs. [41, 34, 33] slightly differ from one another and also from those used in COMPASS analysis.

The average value for the TSA $A_{T}^{\sin \left(2 \varphi_{C S}-\phi_{S}\right)}$ is measured to be below zero with a significance of about two standard deviations. The obtained magnitude of the asymmetry is in agreement with the model calculations of Ref. [42] and can be used to study the universality of the nucleon transversity function. The TSA $A_{T}^{\sin \left(2 \varphi_{C S}+\phi_{S}\right)}$, which is related to the nucleon pretzelos- 
ity TMD PDFs, is measured to be above zero with a significance of about one standard deviation. Since both $A_{T}^{\sin \left(2 \varphi_{C S}-\phi_{S}\right)}$ and $A_{T}^{\sin \left(2 \varphi_{C S}+\phi_{S}\right)}$ are related to the pion Boer-Mulders PDFs, the obtained results may be used to study this function further and to possibly determine its sign. The remaining two asymmetries are the $A_{T}^{\sin \left(\varphi_{C S}-\phi_{S}\right)}$ and $\left.A_{T}^{\sin \left(\varphi_{C S}+\phi_{S}\right)}\right)$ subleading-twist TSAs. Both amplitudes are found to be compatible with zero (see Fig. 9), which can be attributed to the subleading nature of the effects and corresponding dynamic suppressions.

\section{Conclusions}

In this Letter recent COMPASS results of SIDIS measurements of the longitudinal- and transverse-spindependent asymmetries are presented. Compared to the similar studies published by the HERMES and CLAS collaborations, COMPASS results are characterized by an unprecedented precision, covering much wider kinematic range. The two-dimensional representations of COMPASS SIDIS TSAs presented in this

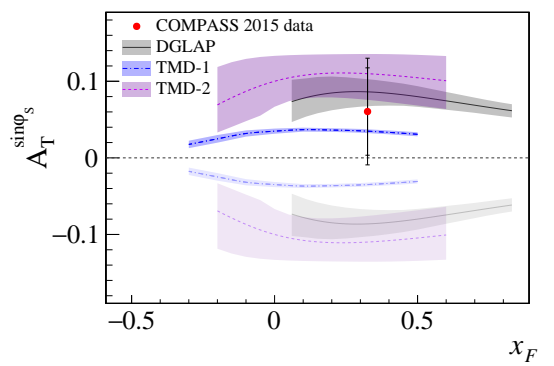

Figure 10: The measured mean Sivers asymmetry and the theoretical predictions from Refs. [41] (DGLAP), [34] (TMD1) and [33] (TMD2). The dark-shaded (light-shaded) predictions are evaluated with (without) the signchange hypothesis Letter are the best currently available input from fixedtarget experiments.

Presented COMPASS Drell-Yan results are the first and the only currently available data on the transverse-spin-dependent azimuthal asymmetries in the Drell-Yan process. In 2018, COMPASS will continue the measurements of the polarized Drell-Yan process for another data-taking year. This will considerably improve the statistical precision of the Sivers and other azimuthal DY TSAs presented in this Letter. Combined with available SIDIS data, COMPASS DY results serve as a crucial input for study of universality of TMD PDFs and for general understanding of the transverse spin structure of the nucleon.

\section{References}

[1] A. Kotzinian, New quark distributions and semiinclusive electroproduction on the polarized nucleons, Nucl. Phys. B441 (1995) 234-248, [hep-ph / 9412283$].$

[2] A. Bacchetta, M. Diehl, K. Goeke, A. Metz, P. J. Mulders and M. Schlegel, Semi-inclusive deep inelastic scattering at small transverse momentum, JHEP 02 (2007) 093, [hep-ph/ 0611265$].$

[3] J. Collins, Foundations of perturbative QCD. Cambridge University Press, 2013.

[4] M. Grosse Perdekamp and F. Yuan, Transverse Spin Structure of the Nucleon, Ann. Rev. Nucl. Part. Sci. 65 (2015) 429-456, [1510.06783].

[5] M. Boglione and A. Prokudin, Phenomenology of Transverse Spin: Past, Present and Future, 1511.06924.

[6] C. A. Aidala, S. D. Bass, D. Hasch and G. K. Mallot, The Spin Structure of the Nucleon, Rev. Mod. Phys. 85 (2013) 655-691, [1209.2803]. 
[7] M. Diehl and S. Sapeta, On the analysis of lepton scattering on longitudinally or transversely polarized protons, Eur. Phys. J. C41 (2005) 515-533, [hep-ph/0503023].

[8] S. Wandzura and F. Wilczek, Sum Rules for Spin Dependent Electroproduction: Test of Relativistic Constituent Quarks, Phys. Lett. B72 (1977) 195-198.

[9] Z. Lu, Single-spin asymmetries in electroproduction of pions on the longitudinally polarized nucleon targets, Phys. Rev. D90 (2014) 014037, [1404.4229].

[10] M. Anselmino, A. Efremov, A. Kotzinian and B. Parsamyan, Transverse momentum dependence of the quark helicity distributions and the Cahn effect in double-spin asymmetry A(LL) in Semi Inclusive DIS, Phys. Rev. D74 (2006) 074015, [hep-ph/ 0608048 ].

[11] S. Boffi, A. V. Efremov, B. Pasquini and P. Schweitzer, Azimuthal spin asymmetries in light-cone constituent quark models, Phys. Rev. D79 (2009) 094012, [0 903.1271$].$

[12] D. Gutierrez-Reyes, I. Scimemi and A. A. Vladimirov, Twist-2 matching of transverse momentum dependent distributions, Phys. Lett. B769 (2017) 84-89, [1702.06558].

[13] A. Kotzinian, B. Parsamyan and A. Prokudin, Predictions for double spin asymmetry A(LT) in semi inclusive DIS, Phys. Rev. D73 (2006) 114017, [hep-ph/ 0603194 ].

[14] D. W. Sivers, Single Spin Production Asymmetries from the Hard Scattering of Point-Like Constituents, Phys. Rev. D41 (1990) 83.

[15] J. C. Collins, Leading twist single transverse-spin asymmetries: Drell-Yan and deep inelastic scattering, Phys. Lett. B536 (2002) 43-48, [hep-ph/ 0204004 ].

[16] S. J. Brodsky, D. S. Hwang and I. Schmidt, Final state interactions and single spin asymmetries in semiinclusive deep inelastic scattering, Phys. Lett. B530 (2002) 99-107, [hep-ph/0201296].

[17] S. J. Brodsky, D. S. Hwang and I. Schmidt, Initial state interactions and single spin asymmetries in Drell-Yan processes, Nucl. Phys. B642 (2002) 344-356, [hep-ph/ 020625 9].

[18] COMPASS COLlabORATION collaboration, P. Abbon et al., The COMPASS experiment at CERN, Nucl. Instrum. Meth. A577 (2007) 455-518, [hep-ex/ 0703049 ].

[19] COMPASS collaboration, F. Gautheron et al., COMPASS-II Proposal, .

[20] S. Arnold, A. Metz and M. Schlegel, Dilepton production from polarized hadron hadron collisions, Phys. Rev. D79 (2009) 034005, [0809.2262].

[21] HERMES collaboration, A. Airapetian et al., Subleading-twist effects in single-spin asymmetries in semi-inclusive deep-inelastic scattering on a longitudinally polarized hydrogen target, Phys. Lett. B622 (2005) 14-22, [hep-ex/ 0505042$].$

[22] CLAS collaboration, H. Avakian et al., Measurement of Single and Double Spin Asymmetries in Deep Inelastic Pion Electroproduction with a Longitudinally Polarized Target, Phys. Rev. Lett. 105 (2010) 262002, [1003.4549].

[23] COMPASS collaboration, C. Adolph et al., Azimuthal asymmetries of charged hadrons produced in high-energy muon scattering off longitudinally polarised deuterons, 1609.06062.

[24] B. Parsamyan, Measurement of longitudinal-target-polarization dependent azimuthal asymmetries in SIDIS at COMPASS experiment, PoS DIS2017 (2018) 259, [1801.01488].

[25] COMPASS COLlaborATION collaboration, M. Alekseev et al., Collins and Sivers asymmetries for pions and kaons in muon-deuteron DIS, Phys. Lett. B673 (2009) 127-135, [0 802 . 2160 ]. 
[26] HERMES COllaboration collaboration, A. Airapetian et al., Observation of the Naive-T-odd Sivers Effect in Deep-Inelastic Scattering, Phys. Rev. Lett. 103 (2009) 152002, [0 906 . 3918].

[27] COMPASS COLLABORATION collaboration, C. Adolph et al., Experimental investigation of transverse spin asymmetries in muon-proton SIDIS processes: Sivers asymmetries, Phys. Lett. B717 (2012) 383-389, [1205.5122].

[28] COMPASS COLLABORATION collaboration, C. Adolph et al., Experimental investigation of transverse spin asymmetries in muon-p SIDIS processes: Collins asymmetries, Phys. Lett. B717 (2012) 376-382, [1205.5121].

[29] COMPASS collaboration, B. Parsamyan, Six 'beyond Collins and Sivers' transverse spin asymmetries at COMPASS, Phys. Part. Nucl. 45 (2014) 158-162, [1301. 6615].

[30] COMPASS collaboration, C. Adolph et al., Collins and Sivers asymmetries in muonproduction of pions and kaons off transversely polarised protons, Phys. Lett. B744 (2015) 250-259, [14 08 . 4405 ].

[31] M. Anselmino, M. Boglione, U. D’Alesio, A. Kotzinian, S. Melis, F. Murgia et al., Sivers Effect for Pion and Kaon Production in Semi-Inclusive Deep Inelastic Scattering, Eur. Phys. J. A39 (2009) 89-100, [0805.2677].

[32] M. Anselmino, M. Boglione and S. Melis, A Strategy towards the extraction of the Sivers function with TMD evolution, Phys. Rev. D86 (2012) 014028, [1204.1239].

[33] P. Sun and F. Yuan, Transverse momentum dependent evolution: Matching semi-inclusive deep inelastic scattering processes to Drell-Yan and W/Z boson production, Phys. Rev. D88 (2013) 114012 , [1308.5003].

[34] M. G. Echevarria, A. Idilbi, Z.-B. Kang and I. Vitev, QCD Evolution of the Sivers Asymmetry, Phys. Rev. D89 (2014) 074013, [1401.5078].

[35] STAR COLlaboration collaboration, L. Adamczyk et al., Measurement of the transverse single-spin asymmetry in $p^{\uparrow}+p \rightarrow W^{ \pm} / Z^{0}$ at RHIC, Phys. Rev. Lett. 116 (2016) 132301, [1511.06003].

[36] COMPASS collaboration, C. Adolph et al., Sivers asymmetry extracted in SIDIS at the hard scale of the Drell-Yan process at COMPASS, 1609.07374.

[37] COMPASS collaboration, M. Aghasyan et al., First measurement of transverse-spin-dependent azimuthal asymmetries in the Drell-Yan process, Phys. Rev. Lett. 119 (2017) 112002, [1704.00488].

[38] B. Parsamyan, First measurement of transverse-spin-dependent azimuthal asymmetries in the Drell-Yan process, PoS DIS2017 (2018) 243, [1801.01487].

[39] H. Avakian, A. V. Efremov, K. Goeke, A. Metz, P. Schweitzer and T. Teckentrup, Are there approximate relations among transverse momentum dependent distribution functions?, Phys. Rev. D77 (2008) 014023, [0 709.3253$]$.

[40] W. Mao, X. Wang, X. Du, Z. Lu and B.-Q. Ma, On the cos $\phi_{h}$ asymmetry in electroproduction of pions in double longitudinally polarized process, Nucl. Phys. A945 (2016) 153-167.

[41] M. Anselmino, M. Boglione, U. D’Alesio, F. Murgia and A. Prokudin, Study of the sign change of the Sivers function from STAR Collaboration W/Z production data, JHEP 04 (2017) 046, [1612.06413].

[42] A. N. Sissakian, O. Yu. Shevchenko, A. P. Nagaitsev and O. N. Ivanov, Polarization effects in Drell-Yan processes, Phys. Part. Nucl. 41 (2010) 64-100. 